\title{
Disparities between IgG4-related disease with and without kidney involvement: a case-control study based on $\mathbf{4 5 0}$ patients
}

\section{Qiaozhu Zeng}

Peking University People's Hospital Department of Rheumatology and Immunology Jingyuan Gao

Affiliated Hospital of North China University of Science and Technology

\section{Xinyu Zhang}

Peking University People's Hospital Department of Radiology

\section{Aichun Liu}

Peking University People's Hospital Department of Nephrology

\section{Zhenfan Wang}

Peking University People's Hospital Department of Rheumatology and Immunology

\section{Ziqiao Wang}

Peking University People's Hospital Department of Rheumatology and Immunology

\section{Xiying Chi}

Peking University People's Hospital Department of Rheumatology and Immunology

\section{Qianyu Shi}

Peking University People's Hospital Department of Rheumatology and Immunology

\section{Yi Wang}

Peking University People's Hospital Department of Rheumatology and Immunology

Yanying Liu ( $\sim$ liuyanying20030801@msn.com )

Peking Universtiy People's Hospital

\section{Zhan-Guo Li}

Peking University People's Hospital Department of Rheumatology and Immunology

\section{Research article}

Keywords: IgG4-related disease, kidney, disparities, risk factor

Posted Date: November 12th, 2020

DOl: https://doi.org/10.21203/rs.3.rs-105954/v1 
License: (c) (i) This work is licensed under a Creative Commons Attribution 4.0 International License. Read Full License 


\section{Abstract}

Background We aimed to compare the demographic, clinical and laboratory characteristics between IgG4related kidney disease (IgG4-RKD+) and extrarenal IgG4-related disease (IgG4-RKD-) in a large Chinese cohort, as well as describing the radiological and pathological features of IgG4-RKD+.

Methods We retrospectively analyzed the medical records of 450 lgG4-RD patients at Peking University People's Hospital from January 2004 to January 2020.

Results Among the 450 IgG4-RD patients, 66 were diagnosed with IgG4-RKD+. Compared with IgG4-RKDpatients, IgG4-RKD+ patients had older age at onset and at diagnosis ( 59 vs 54, $P=0.007 ; 61$ vs 56, $P=0.002$, respectively). Male to female ratio of IgG4-RKD+ patients is significantly higher (2.5:1 vs 1.2:1, $P=0.015)$. In the IgG4-RKD+ group, the most commonly involved organs were salivary gland $(54.6 \%)$, lymph nodes (47.0\%) and pancreas (40.9\%). More than three organs involved (OR=6.212), retroperitoneal fibrosis involvement $(\mathrm{RPF})(\mathrm{OR}=12.460)$ and low serum $\mathrm{C} 3$ level $(\mathrm{OR}=0.202)$ were risk factors for the kidney disease in IgG4-RD patients. It was found that renal function was impaired in more than $50 \%$ of IgG4-RKD+ patients. The most common imaging finding is multiple, often bilateral, hypodense lesions, followed by ureteric obstruction and hydronephrosis related to RPF, and thickening of the renal pelvic wall.

Conclusions Involvement of three or more organs, retroperitoneal fibrosis and low serum C3 level were risk factors for the kidney disease in IgG4-RD patients. These findings indicate potential differences in pathogenesis of these two phenotypes.

\section{Background}

IgG4-related disease (IgG4-RD) is a systemic fibroinflammatory immune-mediated disorder, which is characterized by high levels of serum IgG4, dense lymphoplasmacytic infiltration with IgG4-positive plasma cells in multiple organs, featured storiform fibrosis, and obliterative phlebitis [1-4].

In 2001, IgG4-RD was first described in pancreas, which was called type 1 autoimmune pancreatitis (AIP) [5]. Subsequent studies have revealed that IgG4-RD can affect any organ system. IgG4-related kidney disease (IgG4-RKD+) refers to the renal lesions in association with IgG4-RD. Previous studies have reported the kidney involvement in approximately $12-23 \%$ of total IgG4-RD patients [4, 6-8]. IgG4-RKD + could be manifested by a variety of clinical and histological characteristics, such as tubulointerstitial nephritis (TIN), membranous glomerulonephropathy (MGN) and other infrequent glomerular lesions, pyelitis, hydronephrosis and acute renal failure resulted from retroperitoneal fibrosis (RPF) $[9,10]$.

To minimize the impact of irreversible renal damage resulting from the disease itself or unnecessary surgical intervention, early recognition and treatment are of pivotal importance. In this study, demographic, clinical, and laboratory disparities in the $66 \lg$ G4-RKD + and 384 IgG4RKD- patients were investigated. To our best knowledge, this is the largest study to compare the two phenotypes of patients 
with or without kidney involvement related to IgG4-RD. Determining the characteristics of these two phenotypes may help identify risk factors and their potential differences in pathogenesis.

\section{Methods}

\section{Patients}

From January 2004 to January 2020, four hundred and fifty IgG4-RD patients who were presented at Peking University People's Hospital with complete clinical data were enrolled in this study. Based on the 2011 comprehensive IgG4-RD diagnostic criteria [9], there were 216 (48.0\%), 35 (7.8\%) and 199 (44.2\%) cases diagnosed with definite, probable and possible IgG4-RD, respectively (Table 1). 
Table 1

Demographic, clinical features and laboratory findings of 450 lgG4-RD patients

\section{Characteristics}

\section{Demographics}

Age at disease onset, years, median $55(46,62)$ $(\mathrm{IQR})$

Age at diagnosis, years, median $(\mathrm{IQR})$

Time from onset to diagnosis, years, $\quad 1(0,3)$ median (IQR)

Follow-up period, months, median $(\mathrm{IQR})$

Gender (Male: Female)

Clinical features $\mathrm{n}(\%)$

Complication of allergic disease

Number of involved Organs $\geq 3$

Lymph node

Paranasal sinus

Thyroid gland

Lung

Liver

Pancreas

Biliary system

Gallbladder

Retroperitoneal fibrosis

Mesentery

Aorta

Prostate

Salivary gland
All $(n=450)$

$57(49,64)$

29.8

$(10.9,52.6)$

$1.4: 1$

$185(41.1)$

$293(65.1)$

$205(45.6)$

$61(13.6)$

$46(10.2)$

120 (26.7)

$10(2.2)$

150 (33.3)

76 (16.9)

59 (13.1)

66 (14.7)

$5(1.1)$

15 (3.3)

20 (4.4)

295 (65.6)
IgG4-RKD+ (

$=66$ )

IgG4-RKD- (n $=384$ )

$P$-value

Pvalue 


\begin{tabular}{|c|c|c|c|c|}
\hline Characteristics & All $(n=450)$ & $\begin{array}{l}\text { IgG4-RKD+ (n } \\
=66)\end{array}$ & $\begin{array}{l}\text { IgG4-RKD- (n } \\
=384)\end{array}$ & $P$-value \\
\hline Lacrimal gland & $218(48.4)$ & $25(37.9)$ & $193(50.3)$ & 0.063 \\
\hline Parotid gland & $152(33.8)$ & $20(30.3)$ & $132(34.4)$ & 0.518 \\
\hline \multicolumn{5}{|l|}{ Laboratory findings } \\
\hline Serum IgG4 (mg/dL), median (IQR) & $\begin{array}{l}1030 \\
(348,1660)\end{array}$ & $\begin{array}{l}645 \\
(266,1870)\end{array}$ & $\begin{array}{l}1035 \\
(361,1610)\end{array}$ & 0.753 \\
\hline Serum IgE (IU/ml), median (IQR) & $\begin{array}{l}286.5 \\
(103.4,742.6)\end{array}$ & $\begin{array}{l}211.7 \\
(102.6,501.4)\end{array}$ & $\begin{array}{l}303.9 \\
(116.7,782.4)\end{array}$ & 0.203 \\
\hline $\mathrm{CRP}(\mathrm{mg} / \mathrm{dl})$, median (IQR) & $2.6(1.2,8.2)$ & $4.1(1.4,20.1)$ & $2.4(1.1,7.5)$ & 0.132 \\
\hline $\operatorname{ESR}(\mathrm{mm} / \mathrm{h})$, median $(\mathrm{IQR})$ & $17(8,45)$ & $27.5(11,70)$ & $16(8,38.5)$ & $0.015^{\star \star}$ \\
\hline $\begin{array}{l}\text { Eosinophil count }\left(10^{9} / \mathrm{L}\right) \text {, median } \\
\text { (IQR) }\end{array}$ & $0.2(0.1,0.4)$ & $0.2(0.1,0.3)$ & $0.2(0.1,0.4)$ & 0.627 \\
\hline C3 $(\mathrm{g} / \mathrm{L})$, mean $(\mathrm{SD})$ & $0.9 \pm 0.4$ & $0.8 \pm 0.4$ & $0.9 \pm 0.3$ & 0.053 \\
\hline C4 (g/L), median (IQR) & $0.2(0.1,0.3)$ & $0.2(0.1,0.2)$ & $0.2(0.2,0.3)$ & 0.281 \\
\hline $\mathrm{Hb}(\mathrm{g} / \mathrm{L})$, median (IQR) & $134(121,145)$ & $\begin{array}{l}121.5 \\
(107.3,134.5)\end{array}$ & $\begin{array}{l}135.5 \\
(124,146)\end{array}$ & $0.001^{* *}$ \\
\hline IgG4-RKD + vs IgG4-RKD-, ** $P<0.05$ & & & & \\
\hline
\end{tabular}

Patients were screened for IgG4-RKD + in case of unexplained urinary abnormalities, renal imaging, or histology suggestive of IgG4 RKD + or if they had renal involvement with conditions known to be associated with IgG4-RD. Patients who had any definite etiology of renal impairment were excluded from the study $[11,12]$. Renal pathological findings were available for 6 patients: dense lymphoplasmacytic infiltration with more than 10 infiltrating IgG4-positive plasma cells per high-power field and an $\lg G 4 / \lg G$ plasma cell ratio of more than $40 \%$ with fibrosis. In total, there were 66 patients diagnosed with IgG4RKD+.

Gender, age, allergic diseases, clinical manifestations, organ involvement, radiological and pathological findings were recorded. According to the criteria from the European Academy of Allergy and Clinical Immunology (EAACl), allergic disease was diagnosed by experienced specialists. This study was approved by the Medical Ethics Committee of Peking University People's Hospital (Beijing, China).

\section{Laboratory Features, Imaging Examination And Histological Studies}

We recorded laboratory tests, including serum IgG4 level, serum IgE level, C-reactive protein (CRP), erythrocyte sedimentation rate (ESR), eosinophilia, serum C3, serum $\mathrm{C} 4$, hemoglobulin $(\mathrm{Hb})$, and renal function (including urine specific gravity, $\mathrm{PH}$, proteinuria, urinary white blood cells, urine occult blood, urea 
nitrogen, creatinine, estimated glomerular filtration rate (eGFR), serum $\beta 2$-microglobulin ( $\beta 2-M G)$, urine $N$ acetyl- $\beta$-D-glucosidase(NAG), and retinol-binding protein (RBP)).

All patients underwent radiology examinations consisting of Computed Tomography (CT), or Magnetic resonance imaging (MRI), and some patients also received 18F-fluorodeoxyglucose PET-CT.

We fixed all tissue biopsy samples in formalin and embedded in paraffin, then stained them with hematoxylin and eosin (H\&E) and immunocytochemistry (IHC). IHC was performed using the avidin-biotin complex-peroxidase method with monoclonal antibody to human IgG4 (Zymed, Carlsbad, CA; dilution 1:50 or 1:100 depending on staining laboratory) on sections from paraffin-embedded tissue.

The renal biopsy was examined specifically for features suggestive of IgG4-RKD+, including presence of TIN, lymphoplasmacytic infiltration, as well as tubulointerstitial presentations on light microscopy, glomerular light microscopy abnormalities, glomerular or tubular basement membrane (TBM) deposits on IF or EM. IgG4 staining was done and the number of positive plasma cells/hpf were calculated.

\section{Statistical analysis}

Using Stata software (V.15.0; Stata, College Station, TX, USA), data were analyzed by descriptive methods, with standard summary statistics including mean (S.D.), median, interquartile range (IQR), and proportions. We performed the Student's $t$ test for differences for continuous, normally distributed data; continuous, non-normally distributed data were analyzed by the Mann-Whitney test. Categorical variables were processed by $X^{2}$ or Fisher's exact tests. Logistic regression analysis with enter method was performed to compare the IgG4-RKD + and IgG4-RKD- patients. Factors with $P<0.2$ in the univariate analysis were included in the multivariate analysis. $P<0.05$ was considered to be statistically significant.

\section{Results}

\section{Demographic features}

Demographic details of all the 450 IgG4-RD patients were outlined in Table 1. Among the 450 patients, there were 66 (14.7\%) patients with renal involvement (IgG4-RKD+), and $384(85.3 \%)$ patients without renal involvement (IgG4-RKD-). The comparison of demographic characteristics of IgG4-RKD + and IgG4RKD- patients was also indicated in Table 1. Compared with IgG4-RKD- patients, IgG4-RKD + patients had older age at onset and older age at diagnosis (59 vs $54, P=0.007 ; 61$ vs $56, P=0.002$, respectively). Male to female ratio of IgG4-RKD + patients is significantly higher (2.5:1 vs 1.2:1, $P=0.015)$.

\section{Clinical Characteristics}

As shown in Table 1, there were more patients with involved organs of 3 or more in the IgG4-RKD + group than that in the IgG4-RKD- group ( $84.9 \%$ vs $61.7 \%, P<0.001)$. Detailed organ involvement between IgG4RKD + and IgG4-RKD- groups was shown in Fig. 1. In the IgG4-RKD + group, the most commonly involved organs were salivary gland (54.6\%), lymph nodes (47.0\%) and pancreas $(40.9 \%)$. While in the IgG4-RKD- 
group, salivary gland (67.5\%) was the most commonly affected organ, followed by lacrimal gland (50.3\%), and lymph nodes (45.3\%). The involvement of retroperitoneal fibrosis (36.4\% vs $10.9 \%, P<$ 0.001 ) was more common in IgG4-RKD + patients. While in the IgG4-RKD- group, the involvement of salivary gland $(54.6 \%$ vs $67.5 \%, P=0.042)$ was more common. More patients were significantly associated with allergic diseases in the IgG4-RKD- group than that in the IgG4-RKD + group $(43.2 \%$ vs $28.8 \%, P=0.028)$.

\section{Laboratory Parameters}

Disparities in laboratory features between the IgG4-RKD + and IgG4-RKD- groups were indicated in Table 1. No significant differences were found in levels of serum CRP, IgG4 and IgE, eosinophil count, serum C3, and serum C4. Higher level of ESR was found in the IgG4-RKD + group ( $27.5 \mathrm{vs} 16$ for $\mathrm{Hb}, P=$ 0.015). However, $\mathrm{Hb}$ were significantly lower in the IgG4-RKD + group (121.5 vs $135.5, P=0.001)$.

\section{Renal damage indexes of the lgG4-RKD+ group}

As presented in Table 2, it was found that renal function was impaired with the reduced eGFR (53\%), elevated blood urea nitrogen (37.9\%) and elevated serum creatine (30.3\%). The median eGFR was $78.5 \mathrm{~mL} / \mathrm{min} / 1.73 \mathrm{~m}^{2}(\mathrm{IQR}, 58.5,100.2)$. The level of serum creatinine varied from 64 to $122 \mathrm{umol} / \mathrm{L}$ at baseline. The median blood urea nitrogen was $6.9 \mathrm{mmol} / \mathrm{L}$ (IQR, 5.1, 9.2). Nineteen (28.8\%) of the 66 IgG4-RKD + patients presented with proteinuria ( 1 + or greater on dipstick). Approximately $50 \%$ of IgG4-

RKD + patients presented with urine occult blood. Positive urinary white blood cells (urinary white blood cells $>14 /$ ul) excluding urinary infection were detected in $17(25.8 \%)$ patients. 


\begin{tabular}{|c|c|}
\hline Characteristics & Values \\
\hline Urine specific gravity, median (IQR) & $1.013(1.009,1.020)$ \\
\hline Reduced gravity, n (\%) & $3(4.5)$ \\
\hline PH, median (IQR) & $6(5.5,6.5)$ \\
\hline Reduced PH, n (\%) & $0(0)$ \\
\hline Proteinuria $(1+$ or greater on dipstick), n (\%) & $19(28.8)$ \\
\hline Urine occult blood (+), n (\%) & $32(48.5)$ \\
\hline Urinary white blood cells (+), n (\%) & $17(25.8)$ \\
\hline Estimated GFR, mL/min/1.73 $\mathrm{m}^{2}$ & $78.5(58.5,100.2)$ \\
\hline Reduced estimated GFR (ml/min/1.73m2), n (\%) & $35(53.0)$ \\
\hline Blood urea nitrogen (mmol/L), median (IQR) & $6.9(5.1,9.2)$ \\
\hline Elevated blood urea nitrogen (mmol/L), n (\%) & $25(37.9)$ \\
\hline Serum creatine (umol/L), median (IQR) & $88.5(64,122)$ \\
\hline Elevated serum creatine (umol/L), n (\%) & $20(30.3)$ \\
\hline \multicolumn{2}{|l|}{ Renal tubular function test* } \\
\hline ß2-MG (ug/L), median (IQR) & $2282.5(758,8019.2)$ \\
\hline Elevated $\beta 2-\mathrm{MG}$ (ug/L), n (\%) & $13(92.9)$ \\
\hline Urine N-acetyl- $\beta$-D-glucosidase(NAG) (U/L), median (IQR) & $21.1(14.5,37.3)$ \\
\hline Elevated urine N-acetyl- $\beta$-D-glucosidase(NAG) (U/L), n (\%) & $13(92.9)$ \\
\hline Retinol-Binding Protein (RBP) (mg/L), median (IQR) & $1.2(0.5,2.9)$ \\
\hline Elevated retinol-Binding Protein (RBP) (mg/L), n (\%) & $9(64.3)$ \\
\hline
\end{tabular}

*Fourteen (21.2\%) of the 66 IgG4-RKD+ patients had been tested for renal tubular function.

The renal tubular function was also impaired. Urine specific gravity was reduced in $4.5 \%$ of the 66 patients. Among the 14 IgG4-RKD + patients who had specific renal tubular function tested, the three indexes of $\beta 2-M G, N A G$ and RBP were simultaneously elevated in $9(64.3 \%)$ patients. The median $\beta 2-M G$, NAG and RBP of all the IgG4-RKD patients were 2282.5 ug/L (IQR, 758, 8019.2), 21.1 U/L (IQR, 14.5, 37.3) and $1.2 \mathrm{mg} / \mathrm{L}(\mathrm{IQR}, 0.5,2.9)$, respectively, which were all higher than the normal range.

\section{Risk Factors associated with IgG4-RKD+}

As shown in Table 3, older age at onset ( $\mathrm{OR}=1.028,95 \% \mathrm{Cl}, 1.005-1.051 ; P=0.015)$, older age at diagnosis $(\mathrm{OR}=1.035,95 \% \mathrm{Cl}, 1.011-1.059 ; P=0.004)$, $>=3$ organs involvement $(\mathrm{OR}=3.473,95 \% \mathrm{Cl}$, 1.719-7.020; $P=0.001)$, retroperitoneal fibrosis $(\mathrm{OR}=4.653,95 \% \mathrm{Cl}, 2.566-8.438 ; P<0.001)$, high level of ESR $(\mathrm{OR}=1.014,95 \% \mathrm{Cl}, 1.004-1.023 ; P=0.003)$, low serum C3 levels $(\mathrm{OR}=0.384,95 \% \mathrm{Cl}, 0.164-0.902$; $P=0.028)$ were associated with higher rate of IgG4-RKD + in univariate analysis. Female (OR $=0.498,95 \%$ $\mathrm{Cl}, 0.282-0.881 ; P=0.017)$, allergic disease (OR $=0.531,95 \% \mathrm{Cl}, 0.300-0.939 ; P=0.029)$, salivary gland involvement $(\mathrm{OR}=0.579,95 \% \mathrm{Cl}, 0.341-0.983 ; P=0.043)$, and high level of $\mathrm{Hb}(\mathrm{OR}=0.970,95 \% \mathrm{Cl}$, $0.956-0.985 ; P<0.001)$ were the protective factors of IgG4-RKD+. 
Table 3

Univariate analysis of logistic regression of risk factors for lgG4-RKD + patients

\begin{tabular}{|c|c|c|c|}
\hline \multirow[t]{2}{*}{ Characteristics } & \multicolumn{3}{|c|}{ Univariate analysis } \\
\hline & $P$-value & OR & $95 \% \mathrm{Cl}$ \\
\hline \multicolumn{4}{|l|}{ Demographics } \\
\hline Age at disease onset, median (IQR) & $0.015^{\star \star}$ & 1.028 & $1.005,1.051$ \\
\hline Age at diagnosis, median (IQR) & $0.004 * \star$ & 1.035 & $1.011,1.059$ \\
\hline Time from onset to diagnosis, median (IQR) & 0.860 & 0.994 & $0.927,1.065$ \\
\hline Follow-up period, months, median (IQR) & 0.939 & 1.000 & $0.995,1.005$ \\
\hline Female, n (\%) & $0.017^{\star \star}$ & 0.498 & $0.282,0.881$ \\
\hline \multicolumn{4}{|l|}{ Clinical features } \\
\hline Complication of allergic disease, $\mathrm{n}(\%)$ & $0.029 * *$ & 0.531 & $0.300,0.939$ \\
\hline \multicolumn{4}{|l|}{ Number of involved organs, n (\%) } \\
\hline $1-2$ organs & Ref & Ref & Ref \\
\hline$>=3$ organs & $0.001^{* *}$ & 3.473 & $1.719,7.020$ \\
\hline Lymph node & 0.803 & 1.069 & $0.633,1.804$ \\
\hline Paranasal sinus & 0.425 & 1.336 & $0.655,2.724$ \\
\hline Thyroid gland & 0.582 & 1.256 & $0.558,2.828$ \\
\hline Lung & 0.307 & 1.343 & $0.763,2.367$ \\
\hline Liver & $0.181^{\star}$ & 2.565 & $0.646,10.179$ \\
\hline Pancreas & $0.159 *$ & 1.469 & $0.860,2.509$ \\
\hline Biliary system & 0.762 & 1.111 & $0.563,2.195$ \\
\hline Gallbladder & $0.190 *$ & 1.593 & $0.794,3.196$ \\
\hline Retroperitoneal fibrosis & $<0.001^{\star \star}$ & 4.653 & $2.566,8.438$ \\
\hline Mesentery & 0.736 & 1.462 & \\
\hline Aorta & $0.192^{\star}$ & 2.188 & $0.675,7.088$ \\
\hline Prostate & $0.190 *$ & 2.016 & $0.707,5.749$ \\
\hline Salivary gland & $0.043^{\star *}$ & 0.579 & $0.341,0.983$ \\
\hline Lacrimal gland & $0.065^{\star}$ & 0.603 & $0.353,1.031$ \\
\hline
\end{tabular}




\begin{tabular}{|llll|}
\hline Characteristics & \multicolumn{3}{l|}{ Univariate analysis } \\
\cline { 2 - 4 } & P-value & OR & $95 \% \mathrm{Cl}$ \\
\hline Parotid gland & 0.519 & 0.830 & $0.471,1.461$ \\
\hline Laboratory findings & & & \\
\hline Serum IgG4 (mg/dL), median (IQR) & 0.823 & 1.000 & $1.000,1.000$ \\
\hline Serum IgE (IU/ml), median (IQR) & 0.227 & 1.000 & $0.999,1.000$ \\
\hline $\mathrm{CRP}(\mathrm{mg} / \mathrm{dl})$, median (IQR) & 0.254 & 1.007 & $0.995,1.020$ \\
\hline $\mathrm{ESR}(\mathrm{mm} / \mathrm{h})$, median $(\mathrm{IQR})$ & $0.003^{\star *}$ & 1.014 & $1.004,1.023$ \\
\hline Eosinophil count $(109 / \mathrm{L})$, median $(\mathrm{IQR})$ & $0.145^{\star}$ & 1.342 & $0.903,1.994$ \\
\hline $\mathrm{C} 3(\mathrm{~g} / \mathrm{L})$, mean $(\mathrm{SD})$ & $0.028^{\star *}$ & 0.384 & $0.164,0.902$ \\
\hline $\mathrm{C} 4(\mathrm{~g} / \mathrm{L})$, median $(\mathrm{IQR})$ & 0.362 & 0.409 & $0.060,2.788$ \\
\hline $\mathrm{Hb}(\mathrm{g} / \mathrm{L})$, median $(\mathrm{IQR})$ & $<0.001^{\star *}$ & 0.970 & $0.956,0.985$ \\
\hline$\star P<0.2, * \star P<0.05$ & & & \\
\hline
\end{tabular}

However, only $>=3$ organs involvement $(\mathrm{OR}=6.212,95 \% \mathrm{Cl}, 1.095-35.240 ; P=0.039)$, retroperitoneal fibrosis $(\mathrm{OR}=12.460,95 \% \mathrm{Cl}, 3.080-50.407 ; P<0.001)$ and serum C3 levels $(\mathrm{OR}=0.202,95 \% \mathrm{Cl}, 0.045-$ $0.902 ; P=0.036$ ) remained significant after multivariate analysis (Fig. 2 ).

\section{Pathological findings}

In total, 6 patients with IgG4-RKD underwent renal biopsy; all of the $6(100 \%)$ patients had TIN. TIN in 1 case $(16.6 \%)$ was associated with glomerular disease. Membranous nephropathy was the cause of glomerular disease in this case.

All the patients with TIN had a lymphoplasmocytic (LPC) infiltrate with fibrosis. The LPC infiltration was diffuse in 3 patients and patchy in the other 3 patients. In all of the 6 patients with TIN, IgG4 staining demonstrated > $10 \operatorname{lgG} 4$ + plasma cells per high-power field (in the most concentrated area), and all these patients fulfilled the Raissian criteria for IgG4-TIN [13].

\section{Radiological Findings}

We used contrast-enhanced CT to identify radiological abnormalities in IgG4-RKD + patients except those with renal dysfunction. Sixty-three of the $66 \operatorname{lgG} 4-\mathrm{RKD}+$ patients exhibited characteristic findings of the kidney radiology. Among them, 14 patients were presented with more than one kind of lesion. The most common finding is multiple, often bilateral, hypodense lesions in 32 (48.5\%) IgG4-RKD + patients, which are called small cortical hypodense nodules (Fig. 3A), followed by ureteric obstruction and 
hydronephrosis related to RPF in 22 (33.3\%) patients (Fig. 3B), and thickening of the renal pelvic wall in 18 (27.3\%) IgG4-RKD + patients (Fig. 3C). Besides, diffuse patchy involvement, tumor-like less-enhanced mass and rim-like lesion were observed in 8 (12.1\%), 2 (3.0\%) and 1(1.5\%) patient, respectively.

\section{Discussion}

In this study, we compared the demographic, clinical, and laboratory characteristics of 66 IgG4-RKD+ patients and 384 IgG4-RKD- patients, as well as describing the radiological and pathological findings in patients with IgG4-RKD+. To our best knowledge, this is the largest case-control study of IgG4-RKD+ and IgG4-RKD- phenotypes.

IgG4-RD is manifested by typical clinical features, including tumor-like lesions, dense infiltration with IgG4-positive plasma cells, and extensive fibrosis of multiple organs. There is a great variability of disease manifestations for IgG4-RD, and the identification of different IgG4-RD subgroups is crucial, as a consequence of significant disparities in the characteristics of IgG4-RD regarding different organs $[6,14]$. In our study, the frequency of kidney involvement in IgG4-RD patients was $14.7 \%$, which is lower than that of Japan (23.7\%) and Mexico (24.6\%) [6, 15], but similar to that of UK study [11]. The heterogeneity of IgG4-RKD+ definition and ethnicity in various studies may explain for this diversity. The following organs were found more commonly involved in IgG4-RKD+ patients, including salivary gland, lymph nodes and pancreas. In addition, multi-organ involvement was common in IgG4-RKD+ patients. Therefore, it is necessary to perform a general checkup to obtain a comprehensive view of the patients especially for patients with IgG4-RKD+.

Involvement of three or more organs, retroperitoneal fibrosis and low serum C3 level were risk factors for the kidney disease in IgG4-RD patients. IgG4-RD is a multi-organ immune-mediated condition that could influence almost any organ system in the body. More involved organs may suggest the higher activity of disease. In most cases, IgG4-RKD+ is diagnosed in the context of known extrarenal IgG4-RD or active status of IgG4-RD. With progressive renal decline or detection of characteristic radiological features when evaluating extrarenal IgG4-RD, Kidney involvement became evident $[13,16]$, which may explain for the association between involvement of 3 or more organs and IgG4-RKD+. Additionally, renal involvement may appear as an intrinsic kidney disease (IgG4-RKD+) or as a consequence of ureteric obstruction from retroperitoneal fibrosis (IgG4-RPF). IgG4-RPF often concentrated in the periaortic region and ureters can be entrapped, leading to hydronephrosis and renal injury. Therefore, it is reasonable that retroperitoneal fibrosis is a risk factor for lgG4-RKD+.

There are also some interpretations for the association of low serum C3 levels and IgG4-RKD+. The first descriptions of IgG4 TIN was previously described as "idiopathic hypocomplementemic tubulointerstitial nephritis with extensive tubulointerstitial deposits [17]. Only about 16-34\% of IgG4-RD patients have low serum complement levels, despite that hypocomplementemia is a feature of IgG4-RD. Nevertheless, more than $50 \%$ of patients with active IgG4-TIN have low concentration of complement $[13,16]$. Therefore, hypocomplementemia is considered an important serological feature of the disease [18]. In our study, 
hypocomplemenemia was found in $57.7 \%$ of IgG4-RKD+ patients, which is consistent with previous studies. Wang Rong et al found that complement activation may promote the development of IgG4-RKD+ [19]. Therefore, this study added insights into the hypocomplementemia in IgG4-RKD+ patients. Low serum C3 level was found a risk factor for the development of IgG4-RKD+. Hypocomplementaemia is not characteristic feature of most IgG4-RD patients, which often suggests the existence of IgG4-RKD+, thus scrutiny is necessary.

In previously reported studies, kidney function in IgG4-RKD+ patients varies from normal to renal failure, and the development of renal dysfunction also varies from relatively acute to slowly progressive $[13,16$, $18,20,21]$. In our cohort, the renal function was impaired manifesting as the reduced eGFR, elevated blood urea nitrogen, elevated serum creatine and abnormal specific renal tubular function test. It could be attributed to IgG4-related TIN in the patients, or to the glomerular disease. IgG4-related TIN occurred in the vast majority of IgG4-RKD+ patients, and MGN was reported less than that. Consistent with the rates in previous studies, IgG4-related TIN occurred in all the 6 IgG4-RKD+ patients who had received renal biopsy. In the urinalysis in IgG4-related TIN, we could find typically mild to moderate proteinuria, as well as occasionally the presence of white blood cells [16], which was also consistent with our result. For IgG4RD patients, it is necessary to carry out urine routine test and renal function test (both glomerular and tubular function), in order to timely detect glomerular and renal tubular lesions.

Main abnormalities on renal imaging were revealed in a total of 56 (84.8\%) of IgG4-RKD+ patients: multiple low-density nodules, hydronephrosis and thickening of renal pelvic wall. Similar with previous studies, there were also some other imaging manifestations in our cohort, including diffuse patchy involvement of the bilateral kidneys and rim-like lesion of the kidney [11, 22]. CT was the most common mode of renal imaging, including PET-CT, which is increasingly used. PET-CT could contribute to excluding malignancy with little radiative damage. Moreover, PET-CT is helpful for discovering the involvement of some silent lesions, however, its cost should also be taken into account.

One of the limitations of this study is its retrospective nature, indicating that some affected organs may be neglected, although most patients have undergone general examinations, including FDG-PET. Moreover, only a small number of IgG4-RKD+ cases were diagnosed based on renal biopsy. Compared to biopsy from superficial tissue, there would be higher risk of iatrogenic trauma when patients receive deep kidney biopsy, thus some patients would not accept it.

\section{Conclusion}

In summary, we have specified demographic, clinical, and laboratory differences between IgG4-RKD+ patients and IgG4-RKD. IgG4-RKD+ patients had older age at onset and older age at diagnosis. The male to female ratio of IgG4-RKD+ patients is significantly higher. The most commonly involved organs of IgG4-RKD+ patients were salivary gland, lymph nodes and pancreas. Involvement of three or more organs, retroperitoneal fibrosis and low serum C3 level were risk factors for the kidney disease in IgG4-RD 
patients. These findings indicate potential differences in pathogenesis of these two phenotypes with or without kidney involvement.

\section{List Of Abbreviations}

IgG4-RD, lgG4-related disease

IgG4-RKD, IgG4-related disease

RPF, retroperitoneal fibrosis

AIP, autoimmune pancreatitis

TIN, tubulointerstitial nephritis

MGN, membranous glomerulonephropathy

EAACl, European Academy of Allergy and Clinical Immunology

CRP, C-reactive protein

ESR, erythrocyte sedimentation rate

OR, odd's ratio

$\mathrm{Cl}$, confidence interval

IQR, interquartile range

$\mathrm{Hb}$, hemoglobulin

eGFR, estimated glomerular filtration rate

ß2-MG, $\beta 2$-microglobulin

$N A G$, urine N-acetyl- $\beta$-D-glucosidase

RBP, retinol-binding proteinphosphate

TBM, tubular basement membrane

LPC, lymphoplasmocytic.

\section{Declarations}


Ethics approval and consent to participate According to national regulations and in accordance with the Declaration of Helsinki, data protection authority and medical research ethical committee gave their approval. Written informed consent was obtained from all participants.

Consent for publication Not applicable.

Availability of data and materials The datasets generated and/or analyzed during the current study are not publicly available for ethical reasons, as well as privacy reasons but are available from the corresponding author on reasonable request.

Competing interests The authors declare that they have no competing interests.

Funding This work was supported by Project (RDH2020-03) supported by Peking University People's Hospital Scientific Research Development Funds.

Authors' contributions All authors were involved in drafting the article and revising it critically, and all authors have read and approved the submitted version. ZQZ, GJY, LYY and LZG contributed to the design and conception of the study. LAC, ZXY, CXY, SQY and WZQ were responsible for acquisition of data. WZF and WY performed the statistical analyses and contributed to the interpretation of data. ZQZ was the major contributor in writing the manuscript.

Acknowledgements Thanks to the participants, both patients, and staff for making this study possible.

Correspondence: Yanying Liu, Department of Rheumatology and Immunology, Peking University People's Hospital, Beijing, China 100044

(e-mail: liuyanying20030801@msn.com)

\section{References}

1. Kamisawa T, Zen Y, Pillai S, Stone JH. IgG4-related disease. Lancet. 2014;385(9976):1460-71.

2. Stone JH, Zen Y, Deshpande V. IgG4-related disease. N Engl J Med. 2012;366(6):539-51.

3. Masaki Y, Dong L, Kurose N, Kitagawa K, Morikawa Y, Yamamoto M, et al. Proposal for a new clinical entity, IgG4-positive multiorgan lymphoproliferative syndrome: analysis of 64 cases of IgG4-related disorders. Ann Rheum Dis. 2009;68(8):1310-5..

4. Wallace ZS, Deshpande V, Mattoo H, Mahajan VS, Kulikova M, Pillai S, et al. IgG4-Related Disease: Clinical and Laboratory Features in One Hundred Twenty-Five Patients. Arthritis Rheumatol. 2015;67(9):2466-75.

5. Hamano H, Kawa S, Horiuchi A, Unno H, Furuya N, Akamatsu T, et al. High serum IgG4 concentrations in patients with sclerosing pancreatitis. N Engl J Med. 2001;344(10):732-8.

6. Yamada K, Yamamoto M, Saeki T, Mizushima I, Matsui S, Fujisawa Y, et al. New clues to the nature of immunoglobulin G4-related disease: a retrospective Japanese multicenter study of baseline clinical 
features of 334 cases. Arthritis Res Ther. 2017;19(1):262.

7. Stone JH, Brito-Zerón P, Bosch X, Ramos-Casals M. Diagnostic Approach to the Complexity of IgG4Related Disease. Mayo Clin Proc. 2015;90(7):927-39.

8. Quattrocchio G, Roccatello D. IgG4-related nephropathy. J Nephrol. 2016;29(4):487-93.

9. Umehara H, Okazaki K, Masaki Y, Kawano M, Yamamoto M, Saeki T, et al. Comprehensive diagnostic criteria for IgG4-related disease (IgG4-RD), 2011. Mod Rheumatol. 2012;22(1):21-30.

10. Salvadori M, Tsalouchos A. Immunoglobulin G4-related kidney diseases: An updated review. World J Nephrol. 2018;7(1):29-40.

11. Evans RDR, Cargill T, Goodchild G, Oliveira B, Rodriguez-Justo M, Pepper R, et al. Clinical Manifestations and Long-term Outcomes of IgG4-Related Kidney and Retroperitoneal Involvement in a United Kingdom IgG4-Related Disease Cohort. Kidney Int Rep. 2018;4(1):48-58.

12. Singh N, Nada R, Rawat A, Sharma A, Sinha SK, Ramachandran R, et al. Spectrum of IgG4-related Kidney Disease at a Tertiary Care Center. Indian J Nephrol. 2018;28(3):209-214.

13. Raissian Y, Nasr SH, Larsen CP, Colvin RB, Smyrk TC, Takahashi N ,et al. Diagnosis of IgG4-related tubulointerstitial nephritis. J Am Soc Nephrol. 2011;22(7):1343-52.

14. Liu Y, Xue M, Wang Z, Zeng Q, Ren L, Zhang Y, et al. Salivary gland involvement disparities in clinical characteristics of IgG4-related disease: a retrospective study of 428 patients. Rheumatology (Oxford). 2020;59(3):634-640.

15. Martín-Nares E, Hernandez-Molina G, Rodríguez-Ramírez S, Rivera-Fuentes L, Niño-Cruz JA, NúñezAbreu A, et al. IgG4-related kidney disease: experience from a Mexican cohort. Clin Rheumatol. 2020;39(11):3401-3408.

16. Saeki T, Nishi S, Imai N, Ito T, Yamazaki H, Kawano M, et al. Clinicopathological characteristics of patients with IgG4-related tubulointerstitial nephritis. Kidney Int. 2010;78(10):1016-23.

17. Kambham N, Markowitz GS, Tanji N, Mansukhani MM, Orazi A, D'Agati VD. Idiopathic hypocomplementemic interstitial nephritis with extensive tubulointerstitial deposits. Am J Kidney Dis. $2001 ; 37(2): 388-99$.

18. Kawano M, Saeki T, Nakashima H, Nishi S, Yamaguchi Y, Hisano S, et al. Proposal for diagnostic criteria for IgG4-related kidney disease. Clin Exp Nephrol. 2011;15(5):615-626.

19. Wang R, He D, Zhao L, Liang S, Liang D, Xu F, et al. Role of complement system in patients with biopsy-proven immunoglobulin G4-related kidney disease. Hum Pathol. 2018;81:220-228.

20. Martín-Nares E, Hernandez-Molina G, Rodríguez-Ramírez S, Rivera-Fuentes L, Niño-Cruz JA, NúñezAbreu A, et al. IgG4-related kidney disease: experience from a Mexican cohort. Clin Rheumatol. 2020;39(11):3401-3408.

21. Saeki T, Kawano M. IgG4-related kidney disease. Kidney Int. 2014;85(2):251-7.

22. Kawano M, Saeki T, Nakashima H. IgG4-related kidney disease and retroperitoneal fibrosis: An update. Mod Rheumatol. 2019;29(2):231-239. 
Figures

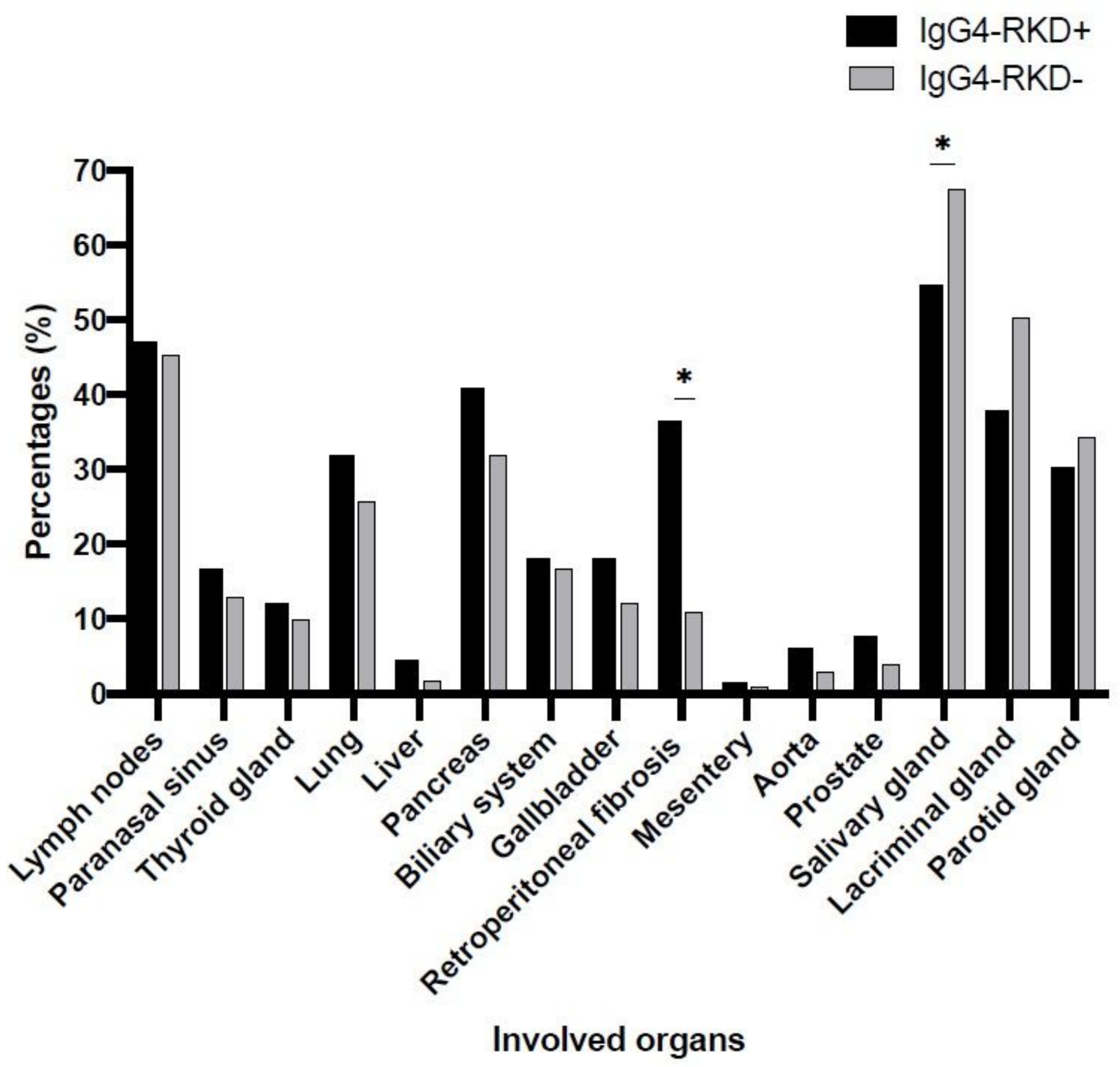

Figure 1

Summary of affected organs in IgG4-RKD+ and IgG4-RKD- patients at baseline. 


\section{Variable}

Age at disease onset

Age at diagnosis

Female

Complication of allergic disease

Involment of $>=3$ organs

Liver

Pamcreas

Gallbladder

Retroperitoneal fibrosis

Aorta

Prostate

Salivary gland

Lacrimal gland

ESR

Eosinophil count

C3

$\mathrm{Hb}$

\section{OR $(95 \% \mathrm{Cl})$}

$1.002(0.9-1.117)$

$1.008(0.895-1.137)$

$0.769(0.265-2.235)$

$0.491(0.17-1.417)$

$6.212(1.095-35.24)$

$0.571(0.049-6.648)$

3.157 (0.996-10.005)

$0.294(0.054-1.6)$

$12.46(3.08-50.407)$

$0.713(0.045-11.275)$

$0.678(0.039-11.7)$

1.303 (0.381-4.459)

$0.944(0.306-2.913)$

1 (0.983-1.018)

$1.385(0.77-2.493)$

$0.202(0.045-0.902)$

$0.992(0.963-1.023)$
$P$-value

0.964

0.892

0.63

0.188

0.039

0.655

0.051

0.157

$<0.001$

0.81

0.789

0.673

0.92

0.993

0.277

0.036

0.622

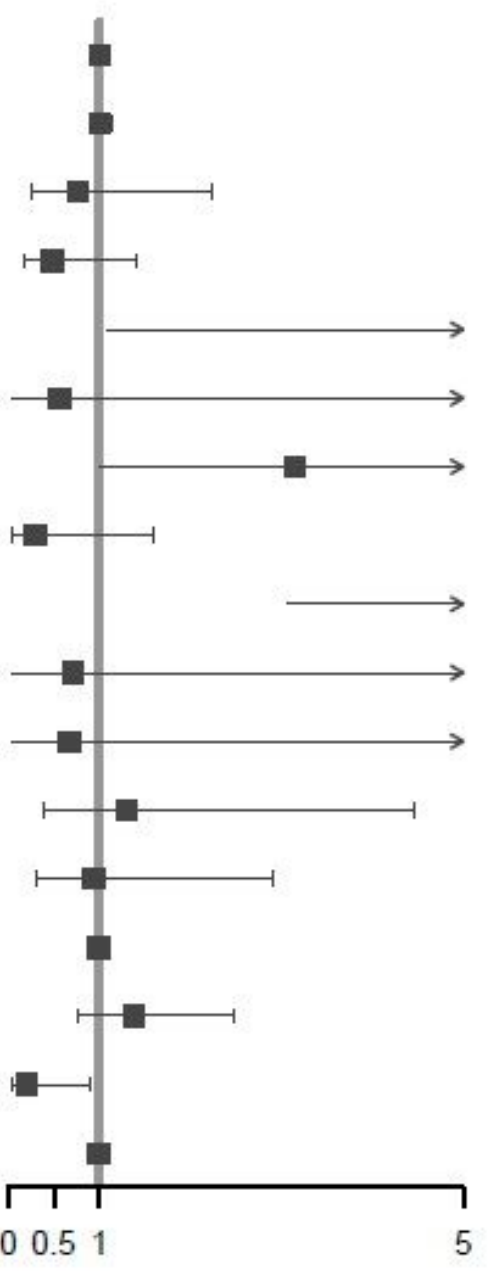

\section{Figure 2}

Multivariate analysis of logistic regression. 

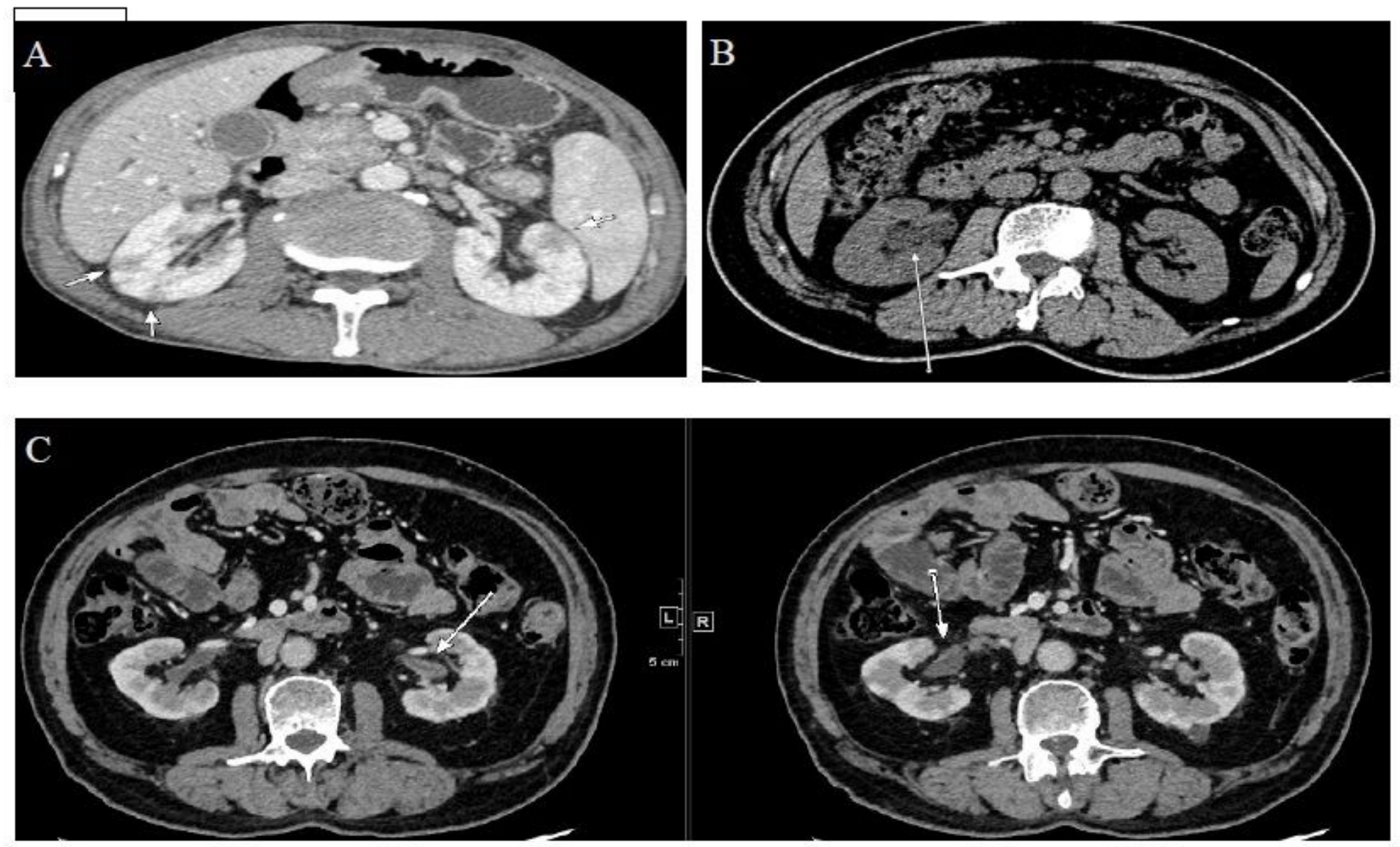

Figure 3

Representative contrast-enhanced CT imaging of IgG4-RKD+. A. Multiple low-density lesions in the bilateral kidneys (arrows). B. ureteric obstruction and hydronephrosis related to RPF (arrow). C. Renal pelvis thickening with smooth intraluminal surface (arrows). 